\title{
MD simulations of the p53 oncoprotein structure: the effect of the Arg273 $\rightarrow$ His mutation on the DNA binding domain
}

\author{
Kholmirzo Kholmurodov ${ }^{1,2 *}$, Ermuhammad Dushanov ${ }^{1,3}$, Kenji Yasuoka ${ }^{4}$ \\ ${ }^{1}$ Laboratory of Radiation Biology, Joint Institute for Nuclear Research, Dubna, Russia; \\ ${ }^{2}$ Dubna University, Dubna, Moscow Region, Russia; \\ ${ }^{3}$ Institute of Nuclear Physics, Tashkent, Uzbekistan; \\ ${ }^{4}$ Department of Mechanical Engineering, Keio University, Yokohama, Japan. \\ E-mail: $\underline{\text { mirzo@jinr.ru }}$
}

Received 26 July 2011; revised 25 August 2011; accepted 15 September 2011.

\begin{abstract}
A comparative molecular dynamics (MD) simulation study was performed on the p53 oncoprotein to investigate the effect of the Arg273His (R273H) mutation on the p53 $\rightarrow$ DNA Binding Domain (DBD). The two p53 dimer structures of the wild-type and mutant Arg273His (R273H) were simulated with the same thermodynamic and environmental parameters. The obtained results demonstrate that the induced Arg273His mutation has a considerable effect on the p53 $\rightarrow$ DNA close contact interaction and changes the picture of hydrogen formation. The Arg273His mutation, in some cases, destroys the existing native hydrogen bond, but, in other cases, forms a strong p53 $\rightarrow$ DNA hydrogen bond, which is not proper for the native protein. The MD simulation results illustrate some molecular mechanism of the conformational changes of the Arg273His key amino acid residue in the p53 $\rightarrow$ DNA binding domain, which might be important for the understanding of the physiological functioning of the p53 protein and the origin of cancer.
\end{abstract}

Keywords: Molecular Dynamics Simulations; p53 Oncoprotein; Effect of the R273H Mutation; DNA Binding Domain

\section{INTRODUCTION}

The p53 tumor suppressor protein is involved in preventing cancer and plays a central role in conserving genomic stability by preventing a genome mutation [1-5]. p53 (the 53 kilodalton ( $\mathrm{kDa}$ ) protein) is activated either to induce a cell cycle arrest allowing the repair and survival of the cell, or apoptosis to discard the damaged cell. Single amino acid substitutions (mutations) in the p53 structure deactivate the p53 protein, which results in cancer [1-8]. Usually, most of the mutations (95\% of all known tumor mutations) occur in the DNA-binding domain (DBD) of the p53 protein. Thus, an oncogenic form of p53 is predominantly a full-length p53 protein with a single amino acid substitution in the DBD. Most of these mutations destroy the ability of the protein to bind to its target DNA sequences, and thus prevent the transcriptional activation of these genes. Tumors with inactive p53 mutants are aggressive and often resistant to ionizing radiation and chemotherapy.

Some reported observations of the effect of the G245S, R248Q, R249S, and R273H mutations suggest that they disturb essentially the stability of the p53 core domain. A detailed analysis of p53 mutations shows that the vast majority of the mutations in p53 cluster in the conserved regions of the DNA-binding core domain (residues 96 292). Around $20 \%$ of all mutations are concentrated in five "hotspot" codons in the core domain: 175, 245, 248, 249, and 273. In [4], examining the Arg273His (R273H) mutation and p53-DNA interactions, it was found that at least three R273H monomers are needed to disable the p53 tetramer. In [6], it is shown that mutations in p53 occur at a rate of approximately $70 \%$ in hormone-refractory prostate cancer and that $\mathrm{R} 273 \mathrm{H}$ p53 mutation (p53R273H) facilitates androgen-independent growth in castrated nude mice, etc.

In studying the p53 protein structural behavior and its mutation transition, molecular dynamics (MD) simulation is a very efficient technique. Nevertheless, few published papers addressed MD simulation of the p53 protein [9-11]. The two major computational difficulties that block the efficient use of MD for the p53 protein could be outlined (as detailed in [9]) as follows: 1) the large size of p53; 2) the problem of accurately modeling the zinc-binding interface in p53c. The p53 protein functions as a tetramer in a cell, but the p53c monomer is already $50 \AA$ in diameter. Simulation of the p53 tetramer 
structure in explicit water is extremely difficult because a large number of water molecules are needed to solvate this protein. The MD simulations performed in [9] were limited to the consideration of the p53c monomer only. It was found that the monomer p53c model is stable in vitro and that such model is still suitable for the protein stability analysis, although the monomer p53c-DNA binding is weaker.

In the present study, we focus on the p53 dimer structure (the A and B chains) to investigate the p53-DNA binding phenomena. The p53 dimer structure is a more adequate model than a monomer one. In the dimer representation, the p53 protein will symmetrically surround the relevant DNA molecule from two sides, which allows the formation of a binding interface in a native manner [12-15]. Next, we perform a comparative MD analysis of the p53 dimer-DNA interaction between the wild-type and mutant Arg273His (R273H) versions of the p53 protein.

\section{MATERIALS AND METHODS}

In Figure 1, the side and top views of the p53 mouse protein are presented (PDB entry file: 3EXJ). For the p53 dimer structure, two chains (A and B) which surround DNA symmetrically are shown along with two catalytic centers [Zn(CYS)3(HIS)1] (the zinc-binding interfaces are displayed in the right pictures; zinc is shown as a gray sphere). On two p53-relevant structures described above (the mouse p53 protein: the wild-type and mutant R273H version (PDB entry file: “3EXJ")), we have performed several model calculations (periodic PMENPT; periodic PME-NVT; non-periodic cutoff; and nonperiodic no-cutoff).

In the p53 protein structure, two chains (A and B) symmetrically surround (yellow arrows) the related DNA sequence located in the central DNA binding domain (DBD). In the p53-DBD interaction, three arginines (R248, R273, and R280), one serine (S241), and one alanine (A276) are responsible for DNA binding. In Figure 1, a positional snapshot of the p53 chain A \{Arg248, Arg273, Arg280, Ser241, Ala276\} with respect to DNA is separately displayed.

Considering the above noted difficulty of zinc binding in p53c, experiments point to the importance of zinc coordination for achieving the correct folding and correct binding of p53 to a specific DNA in intact cells. In our simulation, however, both non-bonded and bonded approaches are used to describe the zinc-p53c binding interface. It should be stressed that it is quite challenging to maintain interface stability at room temperature during long-time simulations [9]. We have followed these criteria and have not fixed zinc locally in its binding interface, even though it is suitable to simulate proteins in which zinc is required for the catalytic function [9]. In this study, we focus on the peculiarities of the global structural changes of the p53 protein dimer. We have performed a comparative analysis of the wild-type and mutant versions between the mouse (mouW, mouM) p53 proteins. For the mouse p53 structures, we have examined the effect of the Arg273His (R273H) mutation on the p53-DNA binding domain.

All simulations were performed with the AMBER (versions 7 to 11) MD software package for studying biomolecules [16,17]. In our periodic MD simulations, the electrostatic interactions were treated with the Particle Mesh Ewald (PME) algorithm [16-18]. Nonperiodic MD involved both cutoff and no-cutoff simulations. To perform no-cutoff calculations, the AMBER

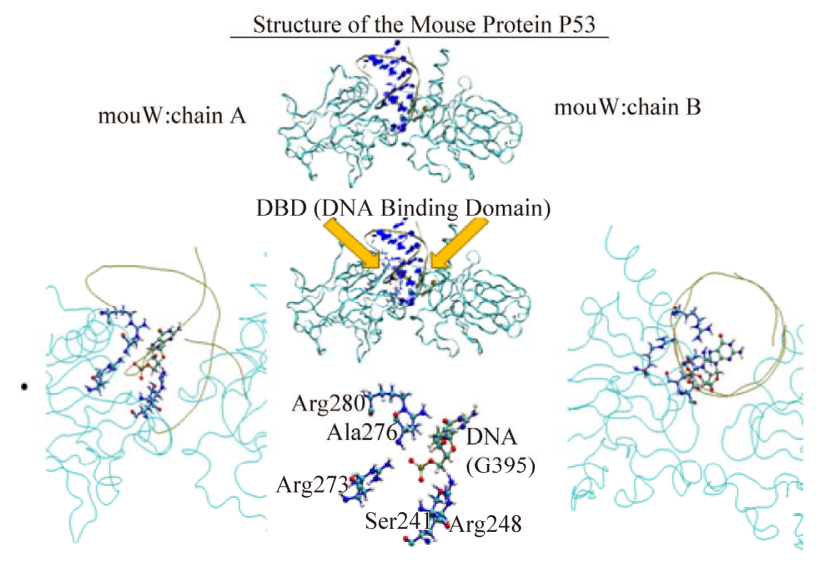

(a)

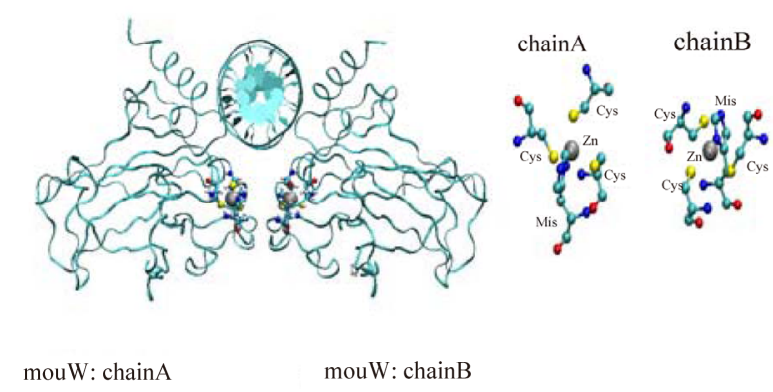

(b)

Figure 1. The side and top views of the p53 mouse protein (PDB entry file: 3EXJ). For the p53 protein structure, two DNA chains (A and $\mathrm{B}$ ) are shown along with two catalytic centers $\left[\mathrm{Zn}(\mathrm{CYS})_{3}(\mathrm{HIS})_{1}\right]$ of the zinc-binding interface (zinc is shown as gray spheres). 
versions were used that had been adapted to the MDGRAPE-2 and 3 hardware. For MDGRAPE-2 and 3, all the particle interactions are calculated [19]. The Cornell et al. all-atom force field was used in the MD simulations [20,21]. A system was solvated with TIP3P molecules [22] generated in a rectangular and spherical (non-periodic) water baths. The temperature was kept constant by using the Berendsen algorithm [23]. Only bond lengths involving hydrogen atoms were constrained using the SHAKE method [24]. The result of simulations and images of the simulated proteins were analyzed using the RasMol [25], MOLMOL [26], and Visual Molecular Dynamics (VMD) [27] software. For some details of our MD simulations, see [28-30]).

\section{Protocol}

Below follows an example of the MD protocol of periodic PME-NPT simulations.

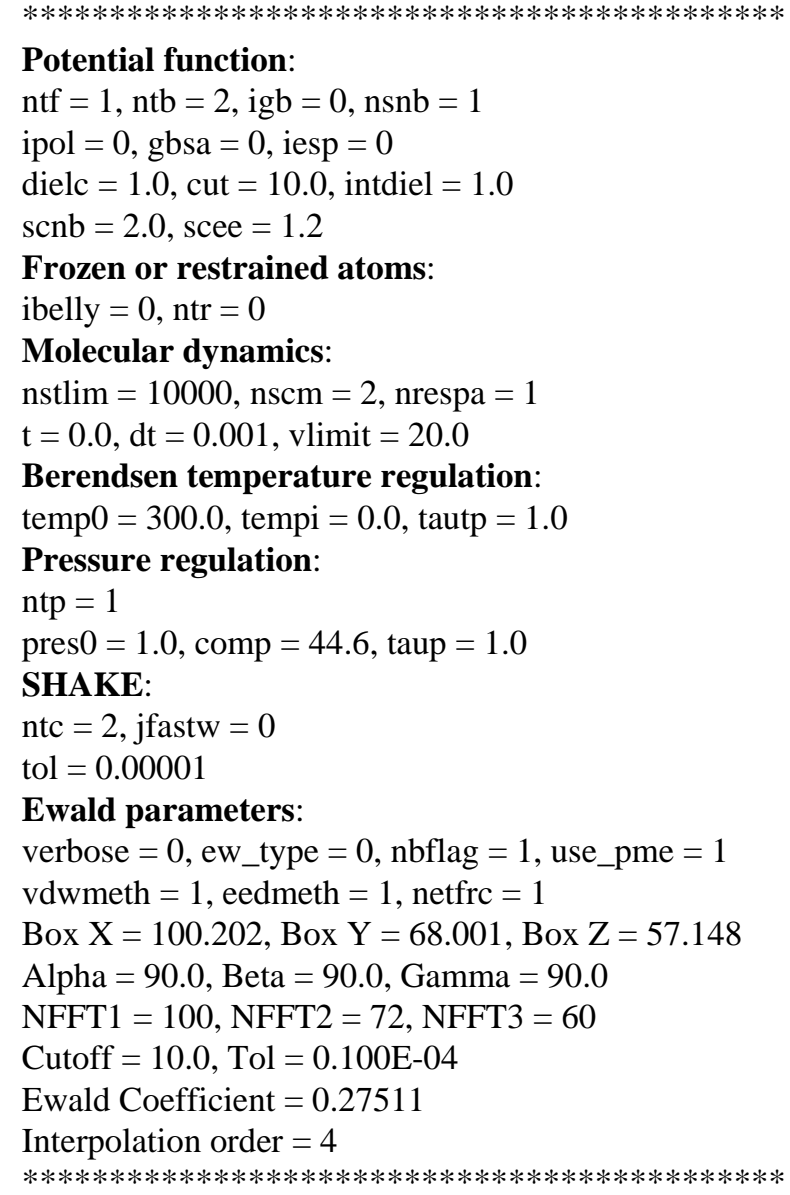

\section{RESULTS AND DISCUSSION}

To compare the conformational changes between the relaxed and original 3D states in the native and mutant structures in Figures 2-5, the side and top views of the p53 mouse protein are shown (PDB entry file: 3EXJ).
We have performed a comparative analysis of the wildtype and mutant versions between the mouse (mouW, mouM) p53 proteins. For the mouse p53 structures, we have examined the effect of the Arg-273His (R273H) mutation on the p53-DNA binding domain.

Arginine (Arg) is an alpha-amino acid with $\mathrm{pKa}=$ 12.48; its side chain consists of a 3-carbon aliphatic straight chain and is positively charged in neutral, acidic, and even most basic environments. Because of conjugation between the double bond and the nitrogen lone pairs, the positive charge is delocalized, enabling the formation of multiple H-bonds. Histidine (His) is an aromatic amino acid with $\mathrm{pKa}=6.5$; its side chain consists of a positively charged imidazole ring which is aromatic at all $\mathrm{pH}$ values. This means that at physiologically relevant $\mathrm{pH}$ values, relatively small shifts in $\mathrm{pH}$ will change its average charge. Below a $\mathrm{pH}$ of 6 , the imidazole ring is mostly protonated [15]. The differences in the chemical structure and properties between arginine and histidine during nanosecond dynamical changes could influence the final (relaxed) states of all amino acids. In Figures 2 and 4, the position of Arg273His $\rightarrow$ DNA for the p53 mouse protein (chain A) are shown at final state $\mathrm{t}=3 \mathrm{~ns}$.

The statistics in Figure 3 (mouW) and Figure 5 (mouM) present the MD calculation results on the positional changes of five amino acid residues related to the direct $\mathrm{p} 53 \rightarrow$ DNA contact:

(mouW)

---top(a) Res $\{$ Arg273 $\} \rightarrow$ DNA $\{$ P(DG395) $\}$

---bottom(b) Res $\{$ Arg273\} $\rightarrow$ DNA $\{$ P(DT394) $\}$

(mouM)

---top(a) Res $\{$ His273 $\} \rightarrow$ DNA $\{$ P(DG395) $\}$

---bottom(b) Res $\{$ His273\} $\rightarrow$ DNA $\{$ P(DT394) $\}$

While constructing the p53 $\rightarrow$ DNA distance diagrams in Figure 3 (mouW) and 5 (mouM), we estimated the distance distributions between the corresponding residue heavy atoms (from the $\mathrm{N}$ - to $\mathrm{CO}$ - ends) and two DNA phosphorus atoms $\mathrm{P}$ (DG395) and P (DT394):

$\mathrm{d}[\operatorname{Res}(\mathrm{N})-\mathrm{DNA}(\mathrm{P})]$, d[Res(CA)-DNA(P)], $\mathrm{d}[\operatorname{Res}(\mathrm{CB})-\mathrm{DNA}(\mathrm{P})], \ldots, \mathrm{d}[\operatorname{Res}(\mathrm{C})-\mathrm{DNA}(\mathrm{P})]$, $\mathrm{d}[\operatorname{Res}(\mathrm{O})-\mathrm{DNA}(\mathrm{P})]$. (The order of the interatomic distances is $\mathrm{d} \sim \AA$ ).

In Figure 3(a) (the top figure), the 3-ns dynamics of the R273 $\rightarrow$ DNA (d[R273-P(DG395)]) interaction distance is shown for the native mouse p53 protein (mouW, chain A). For the wild-type protein, the minimal Arg$273 \rightarrow$ DNA distance is around $3.5 \AA$, which is necessary for p53-DNA bonding. So, p53 forms in this region a close contact with DNA. In Figure 3(b) (the bottom figure), the 3-ns dynamics of the Arg273 $\rightarrow$ DNA (d[R273-P(DT394)]) interaction distance is shown for the mouse p53 protein (mouW, chain A). The minimal distance d[R273-P(DT394)]) increases from 3 - $3.5 \AA$ to $6-7 \AA$. It is clear that in contrast to Figure 3(a), the 


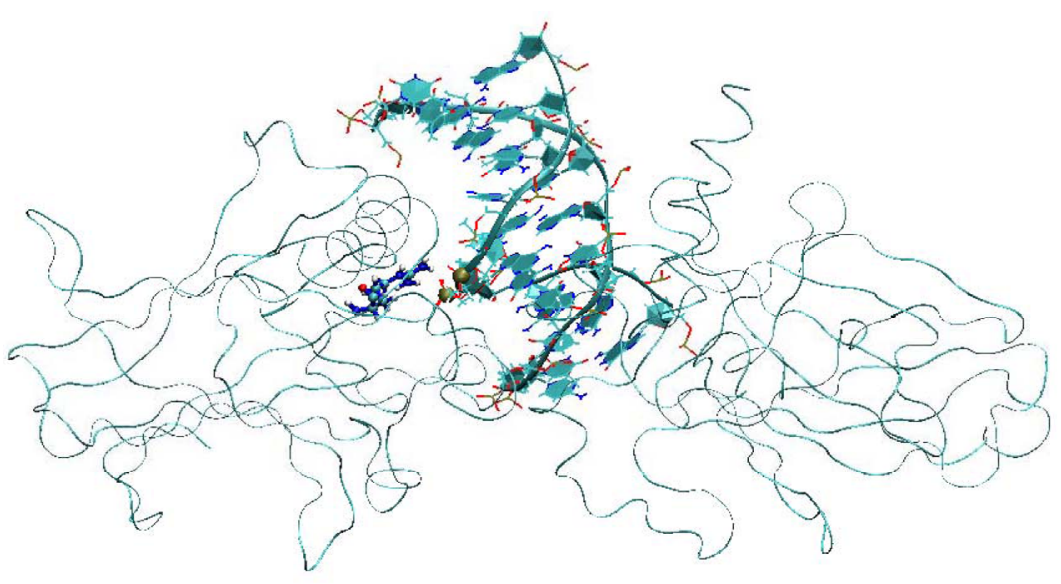

(a)

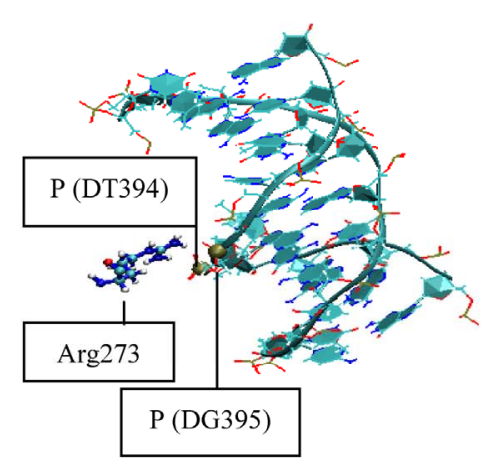

(b)

Figure 2. Snapshots show the amino acid Arg273 $\rightarrow$ DNA contact for the p53 mouse protein (left: chain A; right: chain B). The positions of two closest to Arg273 phosphorus atoms of DNA, P(DG395) and P(DT394), are shown according to the notation of the 3EJX entry file of PDB.

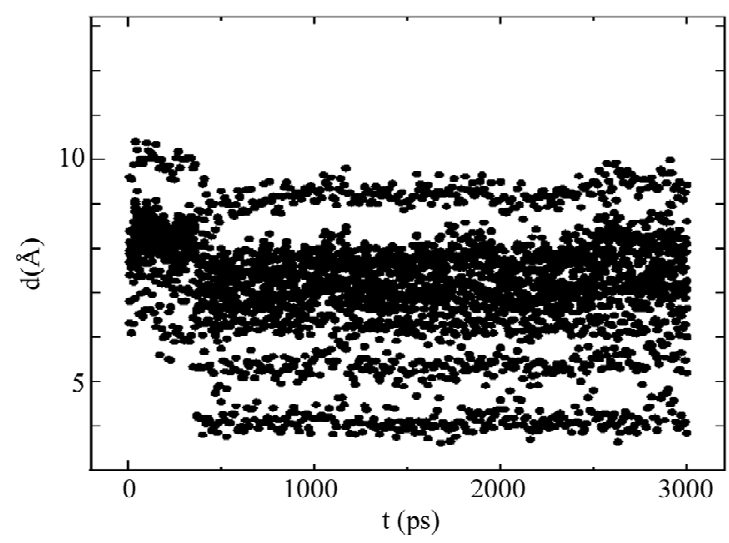

(a)

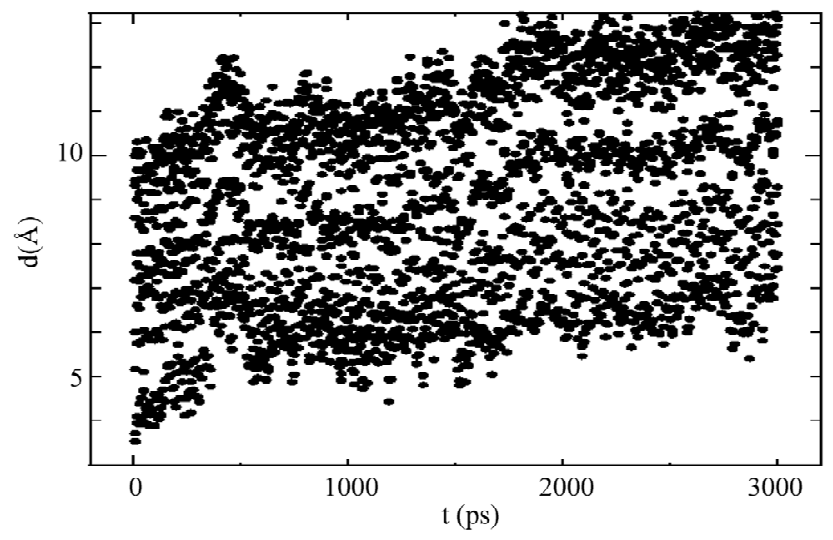

(b)

Figure 3. Distance diagrams of the 3-ns dynamics of the Arg273 $\rightarrow$ DNA contact of the mouse p53 protein (chain A): (a) Arg273-P(DG395); (b) Arg273-P(DT394).

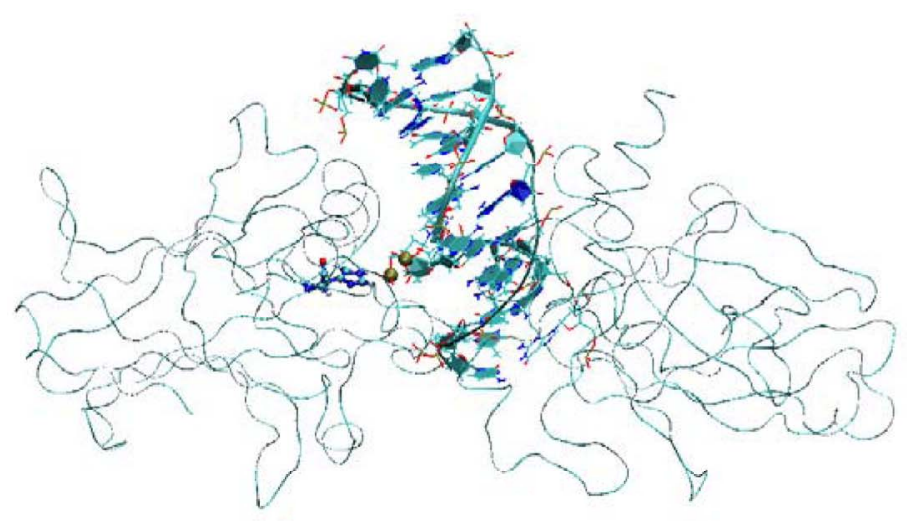

(a)

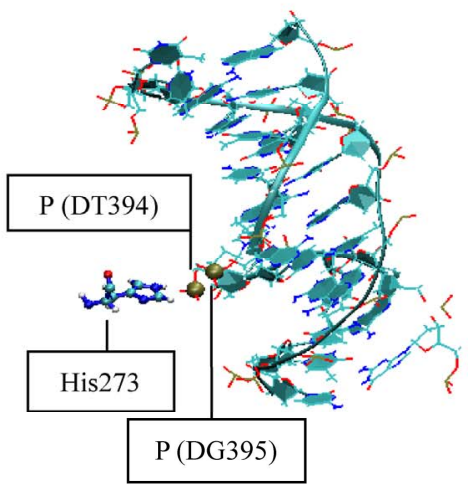

(b)

Figure 4. Snapshots show the amino acid His273 $\rightarrow$ DNA contact for the p53 mouse protein (left: chain A; right: chain B). The positions of two closest to His273 phosphorus atoms of DNA, P(DG395) and P(DT394), are shown according to the notation of the 3EJX entry file of PDB. 


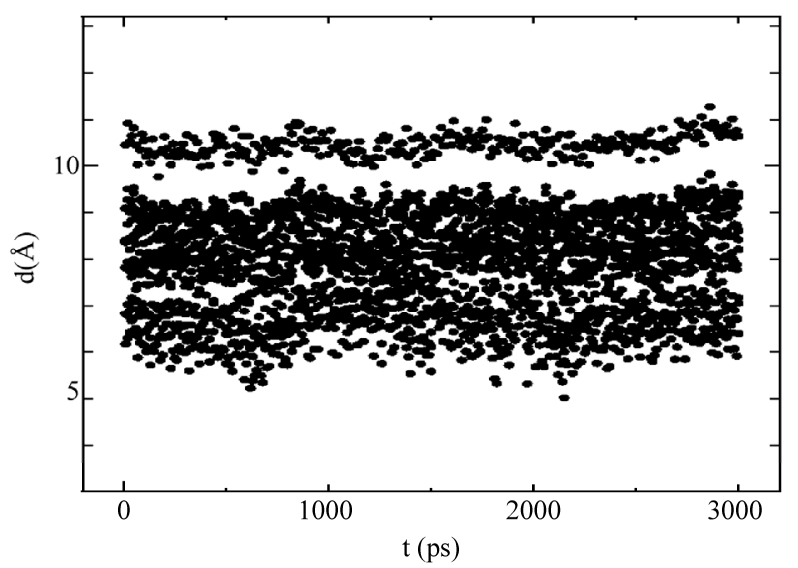

(a)

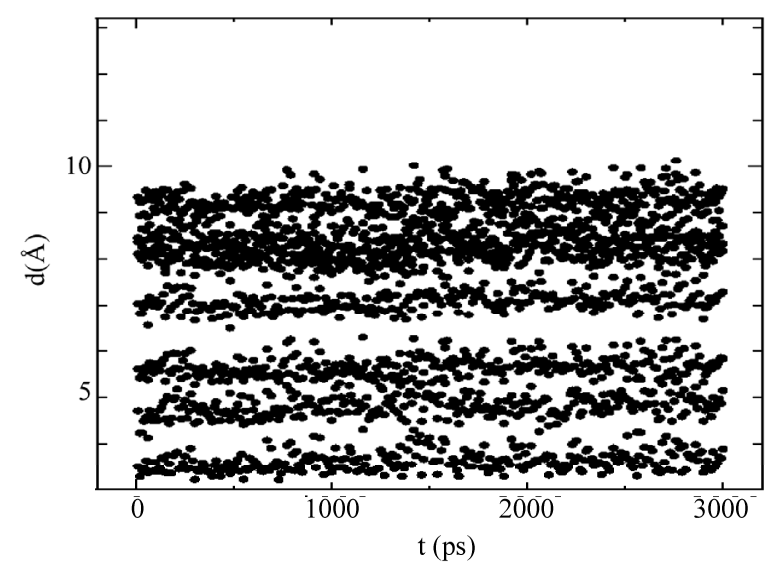

(b)

Figure 5. Distance diagrams of the 3-ns dynamics of the His273 $\rightarrow$ DNA contact of the mouse p53 protein (chain A). (a) His273-P(DG395); (b) His273-P(DT394).

Arg273 is going apart from the $\mathrm{P}(\mathrm{DT} 394)$ atom of the DNA.

In Figure 5(a) (the top figure), the 3-ns dynamics of the His273 $\rightarrow$ DNA (d[H273-P(DG395)]) interaction distance is shown for the mutant p53 protein (mouM, chain A). For the mutant p53 protein, the minimal His $273 \rightarrow$ DNA distance has to be around 6.0 - $6.5 \AA$. Thus, in comparison with Figure 3(a), the induced Arg273His mutation will destroy the p53 $\rightarrow$ DNA close contact. In Figure 5(b) (the bottom figure), the 3-ns dynamics of the His273 $\rightarrow$ DNA (d[H273-P(DT394)]) interaction distance is shown for the mouse p53 protein (mouM, chain A). In this case, for the mutant p53 protein, the minimal His $273 \rightarrow$ DNA distance has to be around 3 - $3.5 \AA$. A comparison between Figures 3(b) and 5(b) is straight-forward.

\section{CONCLUSIONS}

Our MD simulation results on the structure of the p53 oncoprotein with Arg273His (R273H) mutation suggest that the induced mutation disturbs essentially the stability of the p53 core domain. By comparing the relaxed configurations and distance distribution diagrams between the native (Figures $\mathbf{2}$ and $\mathbf{3}$ ) and mutant (Figures $\mathbf{4}$ and 5) p53 proteins, we clearly see that the Arg273His mutation has a considerable effect on the p53 $\rightarrow$ DNA contact. The induced Arg273His mutation essentially changes the picture of hydrogen formation: in some cases, it destroys the existing native hydrogen bond; but, in other cases, it forms a strong p53 $\rightarrow$ DNA hydrogen bond, which is not proper for the native protein. Our MD simulation results illustrate the molecular mechanism of the conformational changes of the Arg273His and key amino acid residue in the p53 $\rightarrow$ DNA binding domain. In conclusion, the obtained results might be important for the understanding of the physiological functioning of the p53 protein and the origin of cancer [1-8].

\section{ACKNOWLEDGEMENTS}

This work has been performed under joint collaboration research between JINR (Russia) and Keio University (Japan). The work has been supported in part by Grant in Aid for the Global Center of Excellence Program for "Center for Education and Research of Symbiotic, Safe and Secure System Design” from Japan's Ministry of Education, Culture, Sport, and Technology. The MD simulations have been performed using computing facilities, software, and clusters at JINR, Russia (CICC); RIKEN, Japan (RICC); RIKEN-Yokohama (MDGARPE-3); and the Yasuoka Laboratory at Keio University, Japan. We thank Prof. Vladimir Korenkov (LIT, JINR) for providing us with computer time at CICC, and Prof. Evgeny Krasavin (LRB, JINR) for his encouraging support.

\section{REFERENCES}

[1] Kern, S.E., Kinzler, K.W., Bruskin, A., Jarosz, D., Friedman, P., Prives, C. and Vogelstein, B. (1991) Identification of p53 as a sequence-specific DNA-binding protein. Science, 252, 170811. doi:10.1126/science.2047879

[2] Maltzman, W. and Czyzyk, L. (1984) UV irradiation stimulates levels of p53 cellular tumor antigen in nontransformed mouse cells. Molecular and Cellular Biology, 4, 168994.

[3] Chumakov, P.M., Iotsova, V.S. and Georgiev, G.P. (1982) Isolation of a plasmid clone containing the mRNA sequence for mouse nonviral T-antigen. Doklady Akademii nauk SSSR, 267, 12725.

[4] Vinall, R.L, Tepper, C.G., Shi, X.-B., Xue, L.A., Gandour-Edwards, R. and De Vere White, R.W. (2006) The R273H p53 mutation can facilitate the androgen-independent growth of LNCaP by a mechanism that involves H2 relaxin and its cognate receptor LGR7. Oncogene, 25, 2082-2093. doi:10.1038/sj.onc.1209246

[5] Joerger, A.C. and Fersht, A.R. (2008) Structural biology of the tumor suppressor p53. Annual Review of Bio- 
chemistry, 77, 557-582. doi:10.1146/annurev.biochem.77.060806.091238

[6] Ma, B. and Levine, A.J. (2007) Probing potential binding modes of the p53 tetramer to DNA based on the symmetries encoded in p53 response elements. Nucleic Acids Research, 35, 7733-7747. doi:10.1093/nar/gkm890

[7] Song, H., Hollstein, M. and Xu, Y. (2007) p53 gainof-function cancer mutants induce genetic instability by inactivating ATM. Nature Cell Biology, 9, 573-580. doi:10.1038/ncb1571

[8] Pan, Y. and Nussinov, R. (2007) Structural basis for p53 binding-induced DNA bending. The Journal of Biological Chemistry, 282, 691-699. doi:10.1074/jbc.M605908200

[9] Lu, Q., Tan, Yu.-H. and Luo, R. (2007) Molecular dynamics simulations of p53 DNA-binding domain. Journal of Physical Chemistry B, 111, 11538-11545. doi:10.1021/jp0742261

[10] Zhou, Z. and Li, Y. (2009) Molecular dynamics simulation of S100B protein to explore ligand blockage of the interaction with p53 protein. Journal of Computer-Aided Molecular Design, 23, 705-714. doi:10.1007/s10822-009-9294-Z

[11] Wang, J., Cao, Z. and Li, S. (2009) Molecular dynamics simulations of intrinsically disordered proteins in human diseases. Current Computer-Aided Drug Design, 5, 280-287. doi:10.2174/157340909789577865

[12] Van Dieck, J., Brandt, T., Teufel, D.P., Veprintsev, D.B., Joerger, A.C. and Fersht, A.R. (2010) Molecular basis of S100 proteins interacting with the p53 homologs p63 and p73. Oncogene, 8, 2024-2035. doi:10.1038/onc.2009.490

[13] Ang, H.C., Joerger, A.C., Mayer, S. and Fersht, A.R. (2006) Effects of common cancer mutations on stability and DNA binding of full-length p53 compared with isolated core domains. Journal of Biological Chemistry, 281, 21934-21941. doi:10.1074/jbc.M604209200

[14] Joerger, A.C., Ang, H.C., Veprintsev, D.B., Blair, C.M. and Fersht, A.R. (2005) Structures of p53 cancer mutants and mechanism of rescue by second-site suppressor mutations. Journal of Biological Chemistry, 280, 1603016037. doi:10.1074/jbc.M500179200

[15] Branden, C. and Tooze, J. (1999) Introduction to protein science. 2nd Edition, Garland Publishing, New York.

[16] Pearlman, D.A., Case, D.A., Caldwell, J.W., Ross, W.R., Cheatham, T.E., DeBolt, S., Ferguson, D., Seibel, G. and Kollman, P. (1995) AMBER, a computer program for applying molecular mechanics, normal mode analysis, molecular dynamics and free energy calculations to elucidate the structures and energies of molecules. Computer Physics Communications, 91, 1995, 1-41. doi:10.1016/0010-4655(95)00041-D

[17] Case, D.C., Pearlman, D.A., Caldwell, J.W., Cheatham III, T.E., Ross, W.S., Simmerling, C.L., Darden, T.A., Merz, K.M., Stanton, R.V., Cheng, A.L., Vincent, J.J., Crowley, M., Ferguson, D.M., Radmer, R.J., Seibel, G.L., Singh, U.C., Weiner, P.K., Kollman, P.A. (2010) AMBER.

[18] Essmann, U., Perera, L., Berkowitz, M.L., Darden, T.,
Lee, H. and Pedersen, L.G. (1995) A smooth particle mesh Ewald method. Journal of Chemical Physics, 103, 8577-8592. doi:10.1063/1.470117

[19] Narumi, T., Susukita, R., Ebisuzaki, T., McNiven, G. and Elmergreen, B. (1999) Molecular dynamics machine: Specialpurpose computer for molecular dynamics simulations. Molecular Simulation, 21, 401-408. doi:10.1080/08927029908022078

[20] Ponder, J.W. and Case, D.A. (2003) Force fields for protein simulations. Advances in Protein Chemistry, 66, 27-85. doi:10.1016/S0065-3233(03)66002-X

[21] Cornell, W.D., Cieplak, P., Bayly, C.I., Gould, I.R., Merz, Jr.K.M., Ferguson, D.M., Spellmeyer, D.C., Fox, T., Caldwell, J.W. and Kollman, P.A. (1995) A second generation forth field for the simulation of Proteins and Nucleic Acids. Journal of the American Chemical Society, 117, 5179-5197. doi:10.1021/ja00124a002

[22] Jorgensen, W.L., Chandrasekhar, J., Madura, J.D. (1983) Comparison of simple potential functions for simulating liquid water. Journal of Chemical Physics, 79, 926-935. doi:10.1063/1.445869

[23] Berendsen, H.J.C., Postma, J.P.M., Van Gunsteren, W.F., DiNola, A. and Haak, J.R. (1984) Molecular dynamics with coupling to an external bath. Journal of Chemical Physics, 81, 3684-3690. doi:10.1063/1.448118

[24] Ryckaert, J.P., Ciccotti, G. and Berendsen, H.J.C. (1997) Numerical integration of the Cartesian equations of proteins and nucleic acids. Journal of Computational Physics, 23, 327-341. doi:10.1016/0021-9991(77)90098-5

[25] Sayle, R.A. and Milner-White, E.J. (1995) RasMol: Biomolecular graphics for all. Trends in Biochemical Sciences, 20, 1995, 374- 376. doi:10.1016/S0968-0004(00)89080-5

[26] Koradi, R., Billeter, M. and Wuthrich, K. (1996) MOLMOL: A program for display and analysis of macromolecular structure. Journal of Molecular Graphics, 4, 1996, 51-55. doi:10.1016/0263-7855(96)00009-4

[27] Humphrey, W., Dalke, A. and Schulten, K. (1996) VMDVisual molecular dynamics. Journal of Molecular Graphics, 14, 33-38. doi:10.1016/0263-7855(96)00018-5

[28] Kholmurodov, K. (Ed.) (2011) Molecular dynamics of nanobistructures. Nova Science Publishers Ltd., Haauppague, 230.

[29] Kholmurodov, K.T., Hirano, Y. and Ebisuzaki, T. (2003) MD simulations on the influence of disease-related amino acid mutations in the human prion protein. Chem-Bio Informatics Journal, 3, 86-95. doi:10.1273/cbij.3.86

[30] Kholmurodov, K., Smith, W., Yasuoka, K., Darden, T. and Ebisuzaki, T. (2000) A smooth-particle mesh Ewald method for DL_POLY molecular dynamics simulation package on the Fujitsu VPP700. Journal of Computational Chemistry, 21, 1187-1191. doi:10.1002/1096-987X(200010)21:13<1187::AID-JCC7 >3.0.CO;2-7 